\title{
In vivo and in vitro estrogenic and progestagenic actions of Tibolone
}

\author{
ANIL SADARANGANI, ANA MARÍA SALGADO, SUMIE KATO, MAURICIO PINTO, \\ ANDRÉS CARVAJAL, CAROLINA MONSO, GARETH I. OWEN and PILAR VIGIL
}

Unidad de Reproducción y Desarrollo, Departamento de Fisiología, Facultad de Ciencias Biológicas, Pontificia Universidad Católica de Chile, Alameda 340, Santiago, Chile.

\begin{abstract}
Estrogen and progestin combination in hormone replacement therapy (HRT) increases the incidence of breast cancer, but decreases the endometrial cancer risk of unopposed estrogen. Therefore, a SERM such as Tibolone, that delivers the beneficial, but not the adverse side effects, of steroid hormones would be clinically advantageous. However, data from the Million Women Study suggests that Tibolone increases the risk of both breast and endometrial cancer. Herein, we assessed the estrogenic and progestagenic actions of Tibolone using transvaginal sonography studies and an in vitro model of breast (ZR-75, MCF7) and endometrial cancer (Ishikawa). The known cancer associated proteins (ER, EGFR, STAT5, tissue factor and Bcl-xL) were selected for study. Transvaginal sonography demonstrated that postmenopausal women treated with Tibolone displayed a thinner endometrium than in the late proliferative phase, but had a phenotype characteristic of the secretory phase, thus demonstrating the estrogenic and progestagenic actions of this SERM. In vitro, Tibolone acted as an estrogen in downregulating ER and upregulating Bcl-xL, yet as progesterone, increasing STAT5 and tissue factor in breast cancer cells. The increase in tissue factor by Tibolone correlated with its coagulative potential. Interestingly, EGFR was up-regulated by progesterone in the breast and by estrogen in endometrial cells, while Tibolone increased protein levels in both cell types. In conclusion, this study further demonstrates the estrogenic and progestagenic nature of Tibolone. The pattern of regulation of known oncogenes in cells of breast and endometrial origin dictates caution and vigilance in the prescription of Tibolone and subsequent patient monitoring.
\end{abstract}

Key words: breast/endometrium cancer, estrogenic, HRT, progestagenic \& tibolone.

\section{INTRODUCTION}

The decrease in hormone production at menopause often leads to climacteric complaints, such as hot flushes and nightsweating. The shortage of sex hormones may also cause the lining of the vagina to become thin and dry (atrophic vaginitis) (1). These physical problems are accompanied in some women by mood changes, nervousness, depression, irritability and loss of sexual desire. Vaginal bleeding may also occur.

A problem which often goes unnoticed is the accelerated bone loss in the years around and after the menopause. As a result of this process the bones become brittle and may easily break (osteoporosis) (2).
Hormone replacement therapy (HRT) is given to alleviate symptoms of estrogenic deficiency; preventing osteoporosis, increasing wellbeing and providing cardiovascular protection. A primary concern with HRT is the incidence of breast cancer observed in the presence of combined estrogen and progesterone therapy. Endometrial proliferation is also a concern during HRT due to an increase risk of endometrial cancer associated to estrogen monotherapy (3). Because the steroidal properties of Tibolone differ from those of conventional HRT, this selective estrogen receptor modulator (SERM) may be a viable and safer alternative for women (4). 
Tibolone (Org OD 14) is a synthetic steroid originally developed by Organon International (Netherlands) that is currently prescribed as an alternative to HRT for postmenopausal women to relieve climacteric symptoms. It has been reported to be metabolized in a tissue-selective manner to three steroids that collectively have weak estrogenic, progestagenic, and androgenic activities (5). Clinically, Tibolone has been shown to relieve climacteric symptoms and prevent bone loss (6). Results of initial studies indicate that Tibolone treatment may also be beneficial for the cardiovascular system (7). Although, these effects are comparable with the effects of estrogen-based HRT, the clinical profile is not exclusively estrogenic. Unlike unopposed estrogen, Tibolone has a less stimulatory effect on post-menopausal endometrium and lower bleeding incidences than estrogen or estrogen plus a progestin. Tibolone therapy improves sexual functioning in postmenopausal women, which implies androgenic action, but it has little androgenic side effects.

When administered orally Tibolone is rapidly metabolized in the liver and intestine or converted locally in target tissues to $3 \alpha$ and $3 \beta$ hydroxy-Tibolone. Both 3-hydroxy metabolites show low affinity binding only to the estrogen receptor (ER), whereas the parent compound Tibolone binds with low affinity to the ER, progesterone (PR) and androgen receptor (AR). Another generated metabolite, a $\Delta 4$-isomer of Tibolone, does not bind to the ER but does possess moderate affinity for the PR and AR. Differences in local metabolism in tissue receptor profiles and differences in hormone responsiveness result in tissue specific effects of Tibolone (8).

The Million Women Study conducted in the United Kingdom between 1996 and 2001 evaluated the role of HRT preparations on the incidence of breast and endometrial cancer (3). This study confirmed previous dogma that unopposed estrogen increased the incidence of endometrial cancer and that combined estrogen and progestin therapy (either cyclic of continuous progestin addition) eliminated the endometrial cancer risk but significantly enhanced the incidence of breast cancer. This study further demonstrated that Tibolone, taken as an alternative to conventional estrogen and progestin preparations, significantly increased the incidence of both breast and endometrial cancer. These clinical findings, coupled to previous in vitro reports, led us to suspect that Tibolone may display estrogenic and progestagenic actions in both a geneand tissue-specific manner. To evaluate this possibility, we selected five proteins known to be regulated by estrogen and progesterone, and compared their regulation in response to Tibolone in a cell line model system of the breast and endometrium.

The ER has been a successful target for effective prevention and treatment strategies in breast cancer, whereas growth factors and their signaling molecules are beginning to be clinically exploited as cancer targets. Understanding the mode of action of Tibolone with respect to the ER and growth factor signaling pathways and their cross-talk with the epidermal growth factor receptor (EGFR) should provide clues needed to optimize treatment approaches and new strategies to overcome and prevent endocrine resistance.

ERs, of which two paralogs have been detected $(\alpha$ and $\beta$ ), are members of the steroid/thyroid hormone superfamily of nuclear receptors (9). The ligand bound estrogen receptor regulates the expression of genes involved in cell proliferation and/ or differentiation. Binding of estrogen (or antiestrogen) to ER causes a conformational change in both receptor types leading to their dimerization, strong association with DNA and recruitment of co-activators or co-repressors. ER $\alpha$ measurement is now routinely used for selecting patients for hormonal therapy at the time of breast cancer diagnosis (10).

STAT (signal transducer and activator of transcription) family members are latent cytoplasmatic proteins that, when activated by phosphorylation, participate in transcripcional regulation in response to various extracellular signals. STAT5 has been shown to regulate growth, 
differentiation and survival of mammary and hematopoietic cells (11). EGFR is a tyrosine kinase receptor of the ErbB family that is abnormally activated in many epithelial tumors. Receptor activation leads to recruitment and phosphorylation of several downstream intracellular substrates, leading to mitogenic signaling and other tumor-promoting cellular activities. In human tumors, receptor over-expression correlates with a more aggressive phenotype and thus poorer patient prognosis (12).

Herein, we also determine the behavior of Tibolone on cancer-markers associated with angiogenesis, metastasis and apoptosis (13). For this purpose we examined the regulation of tissue factor (TF) and $\mathrm{Bcl}-\mathrm{xL}$ by Tibolone. TF is a transmembrane protein responsible for the initiation of the extrinsic coagulation pathway serving as the cofactor and receptor for coagulation Factor VII. The function of TF as initiator of the coagulant pathway can be determined using procoagulant assays. The overexpression of TF is associated with the invasive and metastatic potential of many types of malignancy (14). In breast cancer, elevated TF concentration is correlated with poor prognosis and metastasis $(15,16)$, and a strong relationship has been found between the synthesis of TF and pro-angiogenic indicators such as vascular endothelial growth factor (VEGF) (17). The binding of TF to its ligand FVII provides protection against apoptosis, demonstrating a further potential role of TF in the development and survival of cancer cells (18).

Bcl-xL is an anti-apoptotic member of the Bcl-2 family, which is located mainly on the outer membrane of mitochondria and inhibits a common pathway of apoptosis, at least in part, by preventing the release of cytochrome $c$ into cytosol. The relative ratio of pro- to anti-apoptotic Bcl-2 family members is believed to determine the threshold for induction of mitochondrial-dependent apoptosis. Thus, overexpression of $\mathrm{Bcl}-\mathrm{xl}$ suppresses mitochondrial-mediated apoptosis and enhances cancer cell survival in several cancer cell models (19).

In summary, to determine the behavior of Tibolone we used with patient consent in vivo endometrial diameter studies, coagulation assays and the analysis of five proteins (TF, EGFR, ER $\alpha$, STAT5a/ STAT5b and Bcl-xL) that are known to be associated with tumor development, growth, proliferation, metastasis and angiogenesis. Herein, we demonstrate that Tibolone displays estrogenic and progestagenic behavior in a clinical setting as observed by endometrial diameter examination using ultrasound, and in a gene- and tissue-specific manner in cancer cell lines of breast and endometrial origin.

\section{MATERIALS AND METHODS}

\section{Ultrasound}

Endometrial thickness was evaluated in 15 patients after 3 months of Tibolone use (1.5 $\mathrm{mg} /$ daily or $2.5 \mathrm{mg} /$ daily). Transvaginal sonography was performed using Alpha 2000 GE equipment.

\section{Cell culture and hormonal treatment}

ZR-75 breast cancer cells (20), MCF-7 (21) breast cancer cell lines and Ishikawa endometrial cancer cells (22) were maintained in DMEM/F12 media supplemented with $10 \%$ fetal bovine serum (GibcoBRL). For protein and RNA experiments, cells were plated at $50 \%$ confluence in $10 \mathrm{~cm}^{2}$ Petri dishes (Falcon) and in $6 \mathrm{~cm}^{2}$ Petri dishes for the coagulation assay. The medium was changed to charcoal-treated medium containing 5\% serum for 24 hours before hormone or Tibolone treatment. 17- $\beta$ estradiol (estrogen), progesterone (both SigmaAldrich, St Louis, USA) and Tibolone (Gynopharm, Santiago de Chile) were dissolved in ethanol and added to the cells, individually or in combination, at a final concentration of $10 \mathrm{nM}$.

\section{Western blotting}

Cells were harvested in cold PBS and the pellet resuspendend in lysis buffer $(0.4 \mathrm{M}$ $\mathrm{KCl}, 20 \mathrm{mM}$ Hepes pH 7.4, 1 mM DTT, $20 \%$ glycerol). After sonification on ice, the 
lysate was centrifuged at $14,000 \mathrm{~g}$ for 20 minutes at $4^{\circ} \mathrm{C}$ to separate membrane (pellet) and cytosolic (supernatant) fractions. The crude membrane fraction was resuspended in the above mentioned lysis buffer and protein concentration determined by Bradford assay. One hundred micrograms $(\mu \mathrm{g})$ of crude membrane extract was loaded in each lane, separated by $10 \%$ polyacrylamide gel electrophoresis in the presence of sodium dodecylsulfate, transferred to nitrocellulose membranes, and incubated overnight with specific antibodies. Goat anti-mouse IgG secondary antibody coupled to hydrogen peroxidase (1:5000, Bio-Rad Labs, CA, USA) was applied for one hour at room temperature. Gels were stripped and a-actin or erk2 were applied to confirm equal loading. As for all Western blots shown in this paper, an equal concentration of total protein $(100 \mu \mathrm{g})$ is loaded into each well and confirmed by ponseau staining.

\section{$R T-P C R$}

Total RNA was isolated using the Chomczynski method (23). cDNA was generated using reverse transcriptase (Superscript II, Invitrogen). Using TF primers- Sense:5'-ttc aag aca att ttg gag tgg-3', antisense: 5' -tct cet ggc cca tac act c-3'(BiosChile, Santiago, Chile) semiquantitative PCR reactions were performed from cDNA generated from hormone and EGF treated samples, using Taq polymerase (Invitrogen). Cycle curves were performed for all sets of PCR primers, with the number of cycles used for each primer set being in the linear range of the curve. As an internal control, primers amplifying a region of glyceraldehyde-3-phosphate dehydrogenase (GAPDH) were used. Semiquantitative densitometry of the bands was performed using the NIH Image $1.62 \mathrm{c}$ software package for Macintosh.

\section{Measurement of TF Procoagulant Activity}

TF activity was measured as the ability of cell lysates to accumulate activated factor $\mathrm{X}$ (Xa) in the presence of Factor VIIa. Measurement of TF activity was as follows for ZR-75 and Ishikawa cell lysates. Lysate corresponding to 50,000 cells, in $15 \mathrm{mM} \mathrm{n}$ octyl-beta-D glucopyranoside (SigmaAldrich) was diluted in a solution of $50 \mathrm{mM}$ HEPES buffer, $25 \mathrm{mM} \mathrm{NaCl} 0.1 \%$ and BSA at $\mathrm{pH7.4}$. This mixture was incubated with a reagent mixture containing Factor VIIa (1 $\mathrm{U} / \mathrm{mL})$, factor $\mathrm{X}(1.2 \mathrm{U} / \mathrm{mL})$, and $\mathrm{CaCl} 2(25$ $\mathrm{mM}$; all final concentrations) and Chromozym X (Boehringer Mannheim, 1 $\mathrm{mM})$ in a 96-well plate. Incubation was for 40 minutes at $37^{\circ} \mathrm{C}$ and color development was measured at $405 \mathrm{~nm}$ on a microplate reader (Molecular Devices). Recombinant rabbit Tissue Factor (thromoplastin, Hemoliance Recombiplastin) was used in the construction of a standard curve. Factor $\mathrm{Xa}$ generation, as measured by color change, was converted to $\mathrm{TF}$ procoagulant activity $(\mathrm{U} / \mathrm{mL})$.

\section{RESULTS}

\section{Clinical Observations}

As previous reports have shown, Tibolone effectively reduced climacteric complaints. Although this study by no means constitutes a clinical trial or is it intended to be exhaustive in detail, observations from two years use of Tibolone in our clinic has confirmed the published data that this synthetic steroid displays both estrogenic and progestogenic behaviour. While uterine bleeding is a problem, it is secondary in relation to the benefits delivered. We have consistently noticed that the bleeding observed with Tibolone $(1.5 \mathrm{mg} / \mathrm{day})$ is greater than that observed in women using combined estrogen and progesterone HRT, yet less than observed in premenopausal women using the progesterone-only contraceptives. A similar pattern is observed in regard to the frequency of hot flushes. These observations demonstrate that Tibolone does not display an action specific to one steroid hormone. To determine the effect of Tibolone on the endometrium we used ultrasound to determine endometrial diameter. As demonstrated by five representative ultrasounds in Figure 1, the endometrium 

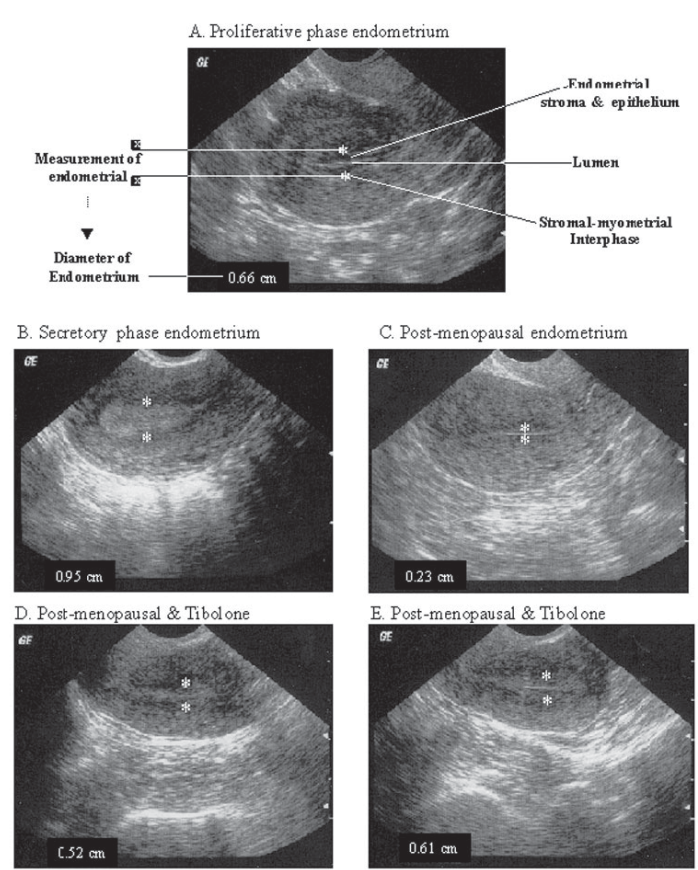

Figure 1. Representative ultrasounds obtained with patient consent of (A) Proliferative phase endometrium, (B) Secretory phase endometrium, (C) Post-menopausal endometrium, (D \& E) Postmenopausal endometrium with Tibolone. The diameter of the endometrium is shown in the lower left side of each panel.

grows in diameter and changes in appearance during the duration of the menstrual cycle. Figure $1 \mathrm{~A}$ shows the endometrium in a late proliferative stage, while in early secretory phase, the diameter of the endometrium has increased considerably and it has taken on a more differentiative appearance as shown in Figure 1B. Figure 1C, demonstrates a postmenopausal endometrium with little or no endometrial development, the mean endometrial thickness at baseline being $0,24 \pm 0.065 \mathrm{~cm}$. However postmenopausal women treated with Tibolone display a thinner endometrium than is found in the late proliferative phase, but possess a phenotype characteristic of the secretory phase (Figures 1D and 1E). The mean endometrial thickness after three months was $0.43 \mathrm{~cm}$. Although the ultrasound images demonstrated in Figures 1D and 1E are at the higher end of the scale, they are shown as clear examples of the secretory phase phenotype. In fact $90 \%$ of women showed an endometrial thickness of less than $0.5 \mathrm{~cm}$. Figures $1 \mathrm{~d}$ and $1 \mathrm{e}$ correspond to women treated with $1.5 \mathrm{mg} / \mathrm{daily}$ Tibolone for an average of three months, however this phenotype is maintained for long periods of time in women receiving Tibolone as HRT. This study was too small to present any significant data on cancer incidence.

\section{Western blot analysis demonstrates that Tibolone exhibits estrogenic and progestagenic behavior in a cell line specific manner.}

As Tibolone demonstrates both estrogenic and progestagenic behavior in the clinic, we chose to use a cell line model system of the breast and endometrium cancer to observe at the level of protein expression the steroid nature of Tibolone. The cell lines chosen for this model were the (ER) and the progesterone receptor (PR) positive MCF-7 and ZR-75 breast cancer cell lines. Both these cell lines are well characterized and have been used previously to identify the steroidal nature of HRT preparations (24). The Ishikawa cell line, derived from an endometrial carcinoma, was selected as our model of the endometrium based on previous reports demonstrating ER and PR positivity and in vivo-like responses to estrogen and progesterone in terms of growth and differentiation (25). As mentioned in the introduction, several candidate proteins were selected for this study based on their previously documented association with sex steroid hormone abnormalities and cancer.

In the MCF-7 breast cell line, western blot analysis demonstrated the expected high levels of ER under basal conditions (control, Figure 2). Furthermore, as anticipated, 24 hours of treatment with 17$\beta$-estradiol reduced ER expression, while progesterone had no effect. Interestingly, treatment with Tibolone for 24 hours reduced ER, to levels lower than that observed with of 17- $\beta$-estradiol. To examine this effect further, we performed a time course of $17-\beta$-estradiol and Tibolone 


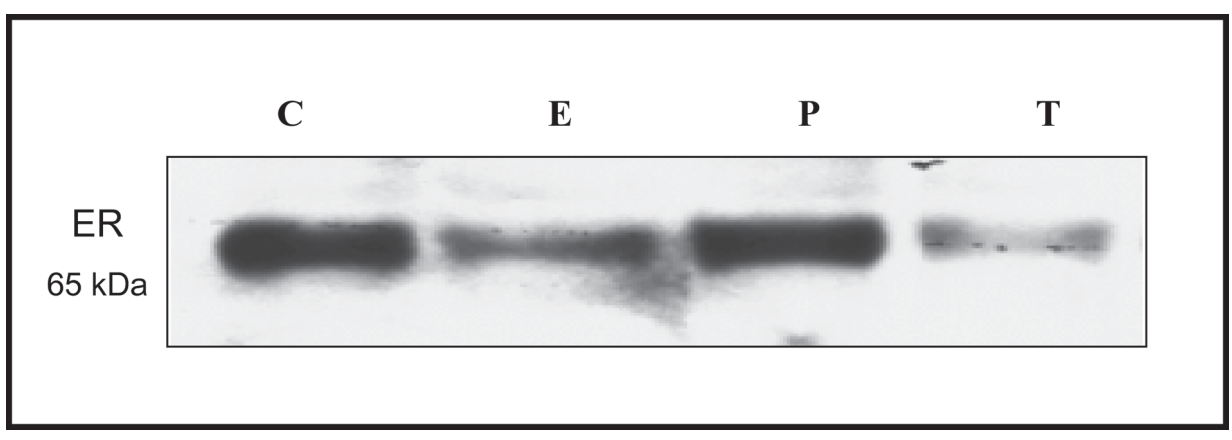

Figure 2. Western blots demonstrating ER expression levels in the MCF-7 cell line, after 24 hours of treatment with Ethanol (C), 17- $\beta$-estradiol (E), progesterone (P) and Tibolone $(\mathrm{T})$.

for 48 hours. Western blot densitometric analysis is demonstrated graphically in Figure 3 . Through negative feedback on its own receptor, 17- $\beta$-estradiol lowers ER levels after 6 and 24 hours of treatment. At 30 hours the ER expression has returned to control levels and at 48 hours these levels have increased further, demonstrating the transient nature of this negative feedback pathway. However, as was observed in Figure 3, Tibolone lowers ER expression at 6 hours, although not to the same extent as the natural ligand, but surprisingly maintains ER levels below basal levels out to 48 hours. Characterization of the estrogen and progesterone half life in this cell system has demonstrated that these steroid hormones can be metabolized and possess a half-life of five to six hours, demonstrating that these cells can rapidly metabolize steroid hormones (results not shown).

Western blot analysis further demonstrated tissue-specific effects of estrogen and progesterone on the regulation of the EGFR (Figure 4). 17- $\beta$-estradiol increased EGFR expression in both the breast and endometrial cells, albeit to a greater extent in the endometrium. Progesterone demonstrates a cell linespecific effect, increasing EGFR expression in the breast cells, while displaying no effect in the endometrium. Interestingly, Tibolone up-regulated the EGF receptor to a greater extent than progesterone in the breast cells (Figure 4A), while demonstrating estrogenic behavior in the endometrial cancer cell line (Figure 4B).

Western blot analysis of a downstream mediator of the cytokines STAT5, is shown in Figure 5 (upper panel). In this Western blot, the upper band corresponds to STAT5a and lower band to STAT5b. No regulation of either isoform is observed in the presence of $17-\beta$-estradiol in ZR-75 cells. However, both progesterone and Tibolone increased expression of both isoforms in the breast cell line. No regulation of the STAT5 isoforms occurred in Ishikawa cells.

The expression of the anti-apoptotic protein, Bcl-xL, is up-regulated in ZR-75 and Ishikawa cells by $17-\beta$-estradiol. No regulation of this protein is observed in the presence of progesterone in either cell line (Figure 5, lower panel). Tibolone displayed cell line-specific behavior, increasing Bcl$\mathrm{xL}$ levels to a greater extent to that of the $17-\beta$-estradiol in ZR-75 levels, yet having no effect in the Ishikawa cell line (lower panel, Figure 5). As a further internal standard of this technique, the second panel of Figure 5 demonstrates that no regulation of the erk-2 protein is observed under hormonal treatments.

Western blot analysis of TF, the initiator of the extrinsic coagulation cascade, reveals the presence of two bands in the ZR-75 breast cancer cell line (Figure 6). We have demonstrated that the lower band corresponds to non-specific cross-reactivity (26). Focusing on TF (glycosylated and 


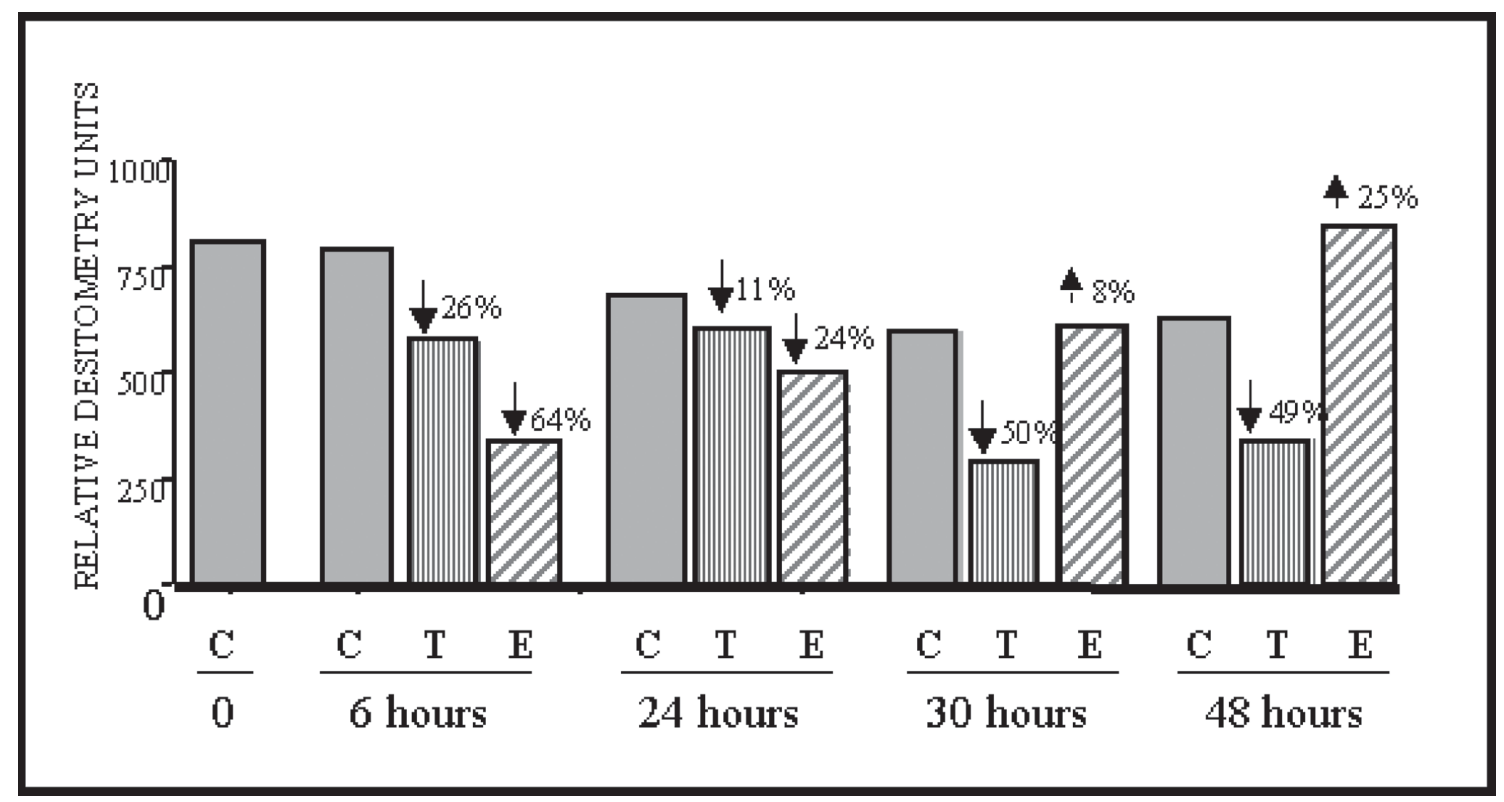

Figure 3. Western blots demonstrating a time course of ER expression in the MCF-7 cell line after the administration of Ethanol (C), 17- $\beta$-estradiol (E) or Tibolone (T).Protein levels are expressed as relative densitometric units. Percentages represent changes in respect to ethanol treatments within each time points.

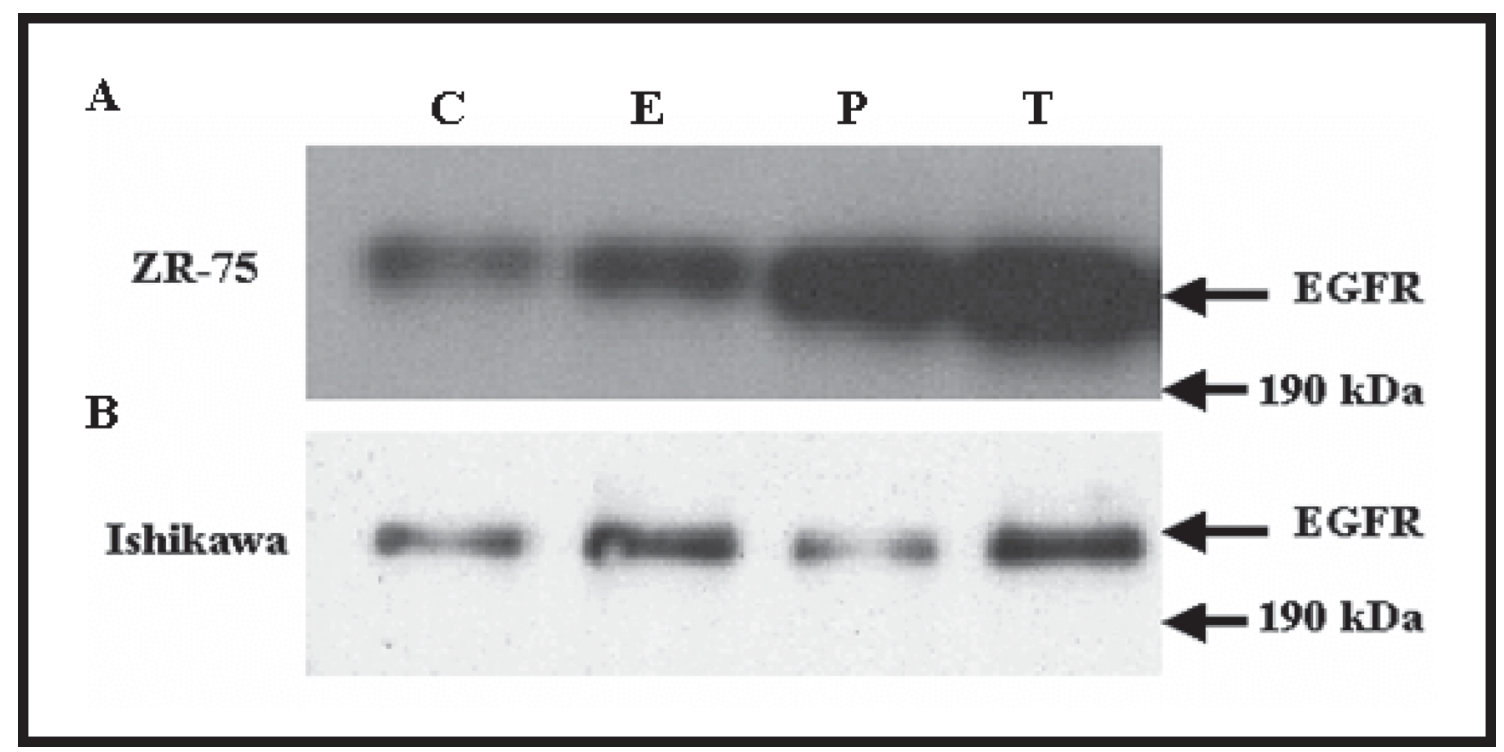

Figure 4. Western blots demonstrating EGFR expression levels after 24 hours of treatment with Ethanol (C), 17- $\beta$-estradiol (E), progesterone (P) and Tibolone (T) in the ZR-75 cell line (Panel A) and the Ishikawa cell line (Panel B). 


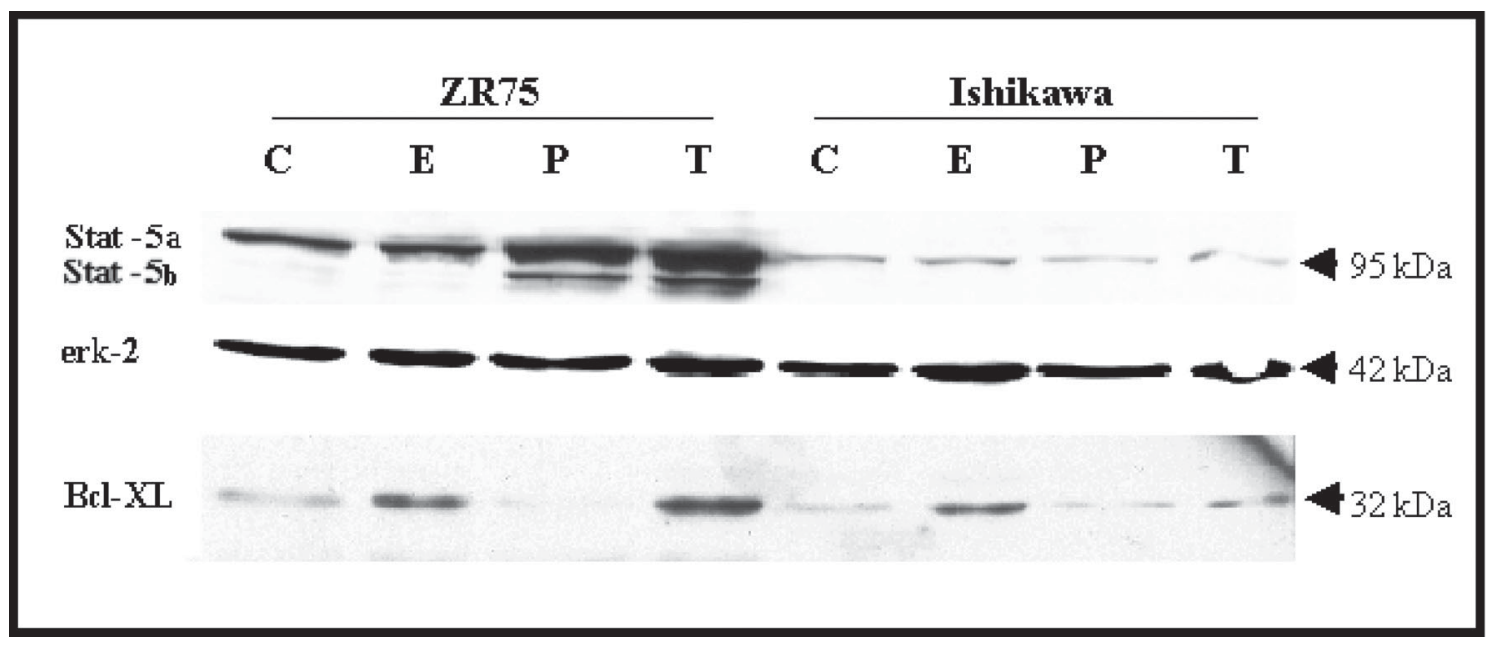

Figure 5. Western blots demonstrating Stat-5a/b (upper panel), erk-2 (internal standard) and Bcl-xL (lower panel) expression levels after the administration of Ethanol (C), 17- $\beta$-estradiol (E), progesterone $(\mathrm{P})$ and Tibolone $(\mathrm{T})$ in $\mathrm{ZR}-75$ and Ishikawa cell lines.

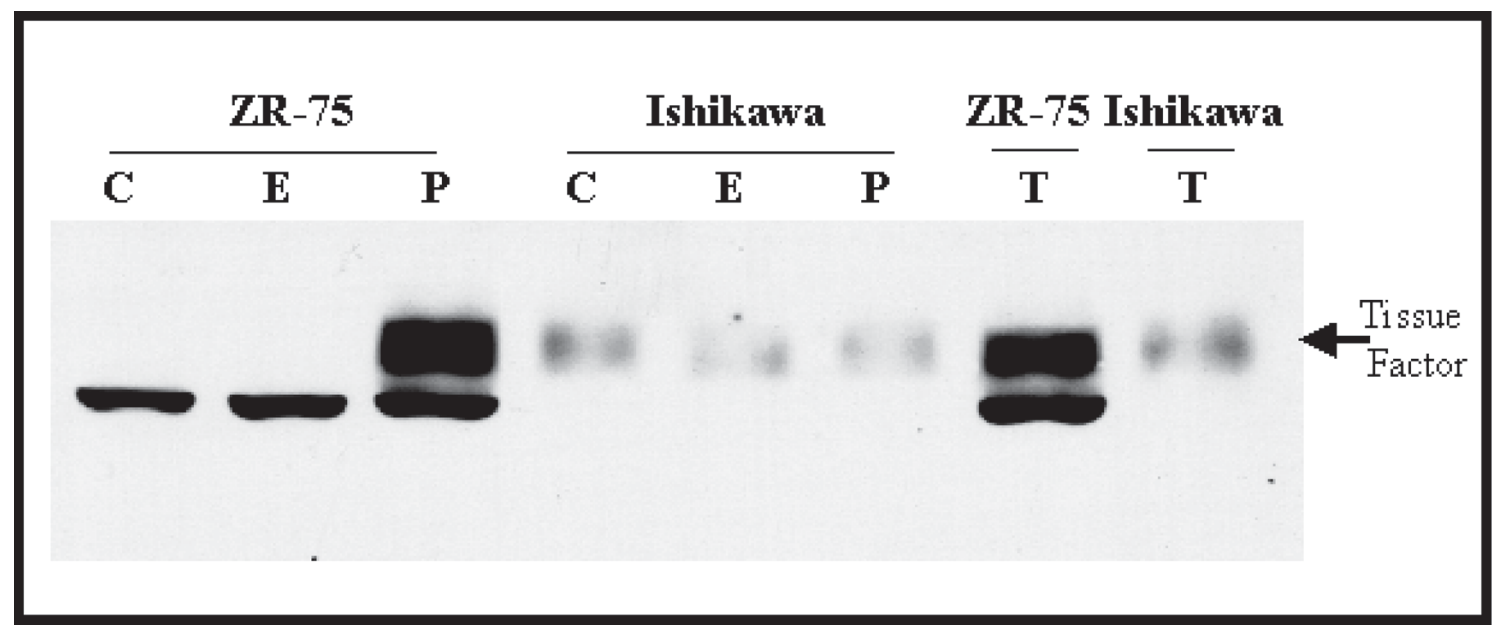

Figure 6. Western blots demonstrating TF expression levels after 24 hours of treatment with Ethanol (C), 17- $\beta$-estradiol (E), progesterone (P) and Tibolone (T) in ZR-75 and Ishikawa cell lines. The lower band in the ZR-75 cell line corresponds to a non-specific band.

thus diffuse upper band), no regulation is observed in the presence of 17- $\beta$-estradiol in either cell line (Figure 6). Progesterone increased TF expression in ZR-75 cells while having no effect in Ishikawa cells. Tibolone exerted an effect similar to that of progesterone, demonstrating an increase in the ZR-75 but not in Ishikawa (Figure 6). To further investigate this observation we performed a time course of progesterone and Tibolone treatment on TF expression in the ZR-75 cell line. As demonstrated in
Figure 7A, progesterone produced a transient increase in $\mathrm{TF}$ expression, being maximum at 24 hours. As observed in Figure 2 with ER, Tibolone maintained elevated TF levels out to 48 hours (Figure 7B).

Tibolone regulates $T F$ expression at the mRNA level.

To examine at which level Tibolone is exerting its regulation of $\mathrm{TF}$ in $\mathrm{ZR}-75$, we 


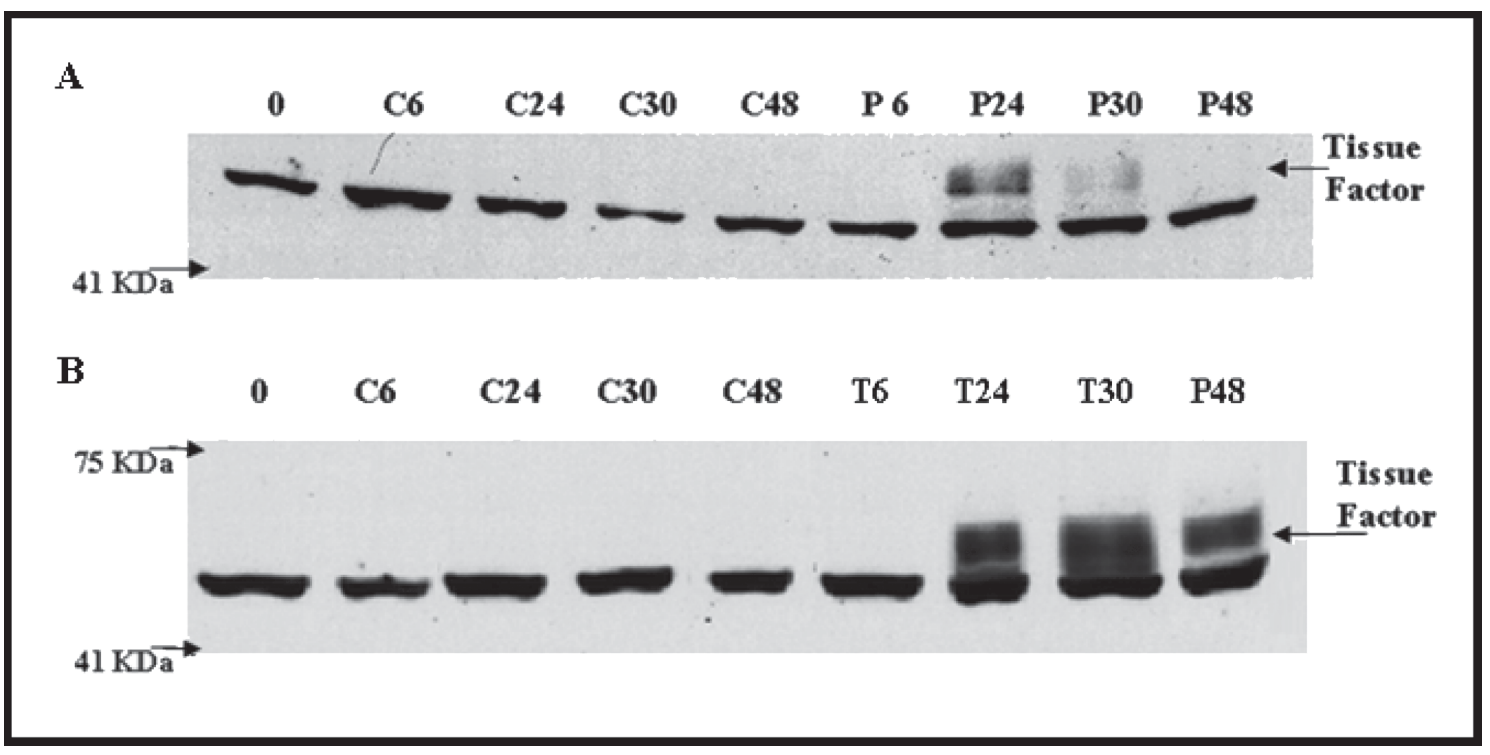

Figure 7. Western blots demonstrating a time course in the ZR-75 cell line of TF expression after 6, 24, 30 and 48 hours of treatment with Ethanol (C) and progesterone (P) in panel A and with Ethanol (C) and Tibolone (T) in panel B. The lower constitutive band corresponds to a non-specific band.

performed RT-PCR to determine if any changes in mRNA were occurring. As shown in Figure 8, Tibolone, along with progesterone, increases the expression of TF mRNA after 9 hours of treatment. GAPDH, which was previously demonstrated not to be under hormonal regulation, was used as an internal loading control. RT-PCR results in the Ishikawa cell line demonstrated, as anticipated, no regulation of TF mRNA under any of the above mentioned treatment conditions (results not shown).

Pro-coagulant activity of TF in ZR-75 and Ishikawa cells

To determine if the induction of TF by progesterone and Tibolone has biological activity and physiological significance, we performed a procoagulant assay in sex steroid hormone-and Tibolone-treated cell line extracts. This coagulation assay determined cell surface TF procoagulant activity as measured by the generation of Factor Xa (see Methods section). As anticipated from western blot analysis, basal procoagulant activity was higher in Ishikawa cells in comparison to ZR-75 cells, reflecting the TF levels observed under basal conditions (compare Figure 9 to control samples in Figure 6). No significant changes in procoagulant activity were observed under any treatment in Ishikawa cells, while both progesterone and Tibolone increased the activity of ZR-75 cell extracts (Figure 9). The levels of TF protein expression, as determined by Western blot analysis in ZR-75 and Ishikawa cells, in the presence and absence of treatment, correlate exactly with the measured procoagulant activity (Figure 9).

\section{DISCUSSION}

Previous studies comparing the effect of Tibolone versus conjugated equine estrogen (CEE) with and without MPA on the reproductive tract of cynomolgus monkeys have shown that endometrial atrophy is found in 29/30 animals receiving a low Tibolone regimen, compared to $23 / 31$ animals with endometrial atrophy receiving a high dose regimen. CEE alone did not induce endometrial atrophy and CEE plus MPA induced it in $11 / 29$ cases. Human studies have shown a minimal increase in 


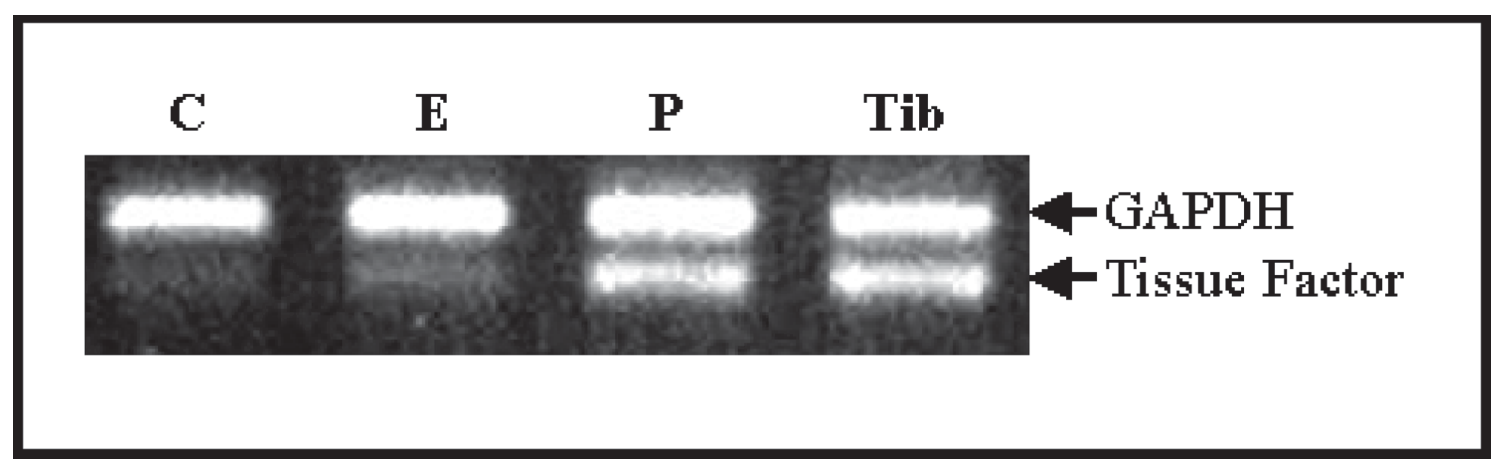

Figure 8. RT-PCR of TF (lower band) and GAPDH (internal loading control, upper band). ZR-75 cells were treated with Ethanol (C), 17- $\beta$-estradiol (E), progesterone (P) and Tibolone (T) for 9 hours.

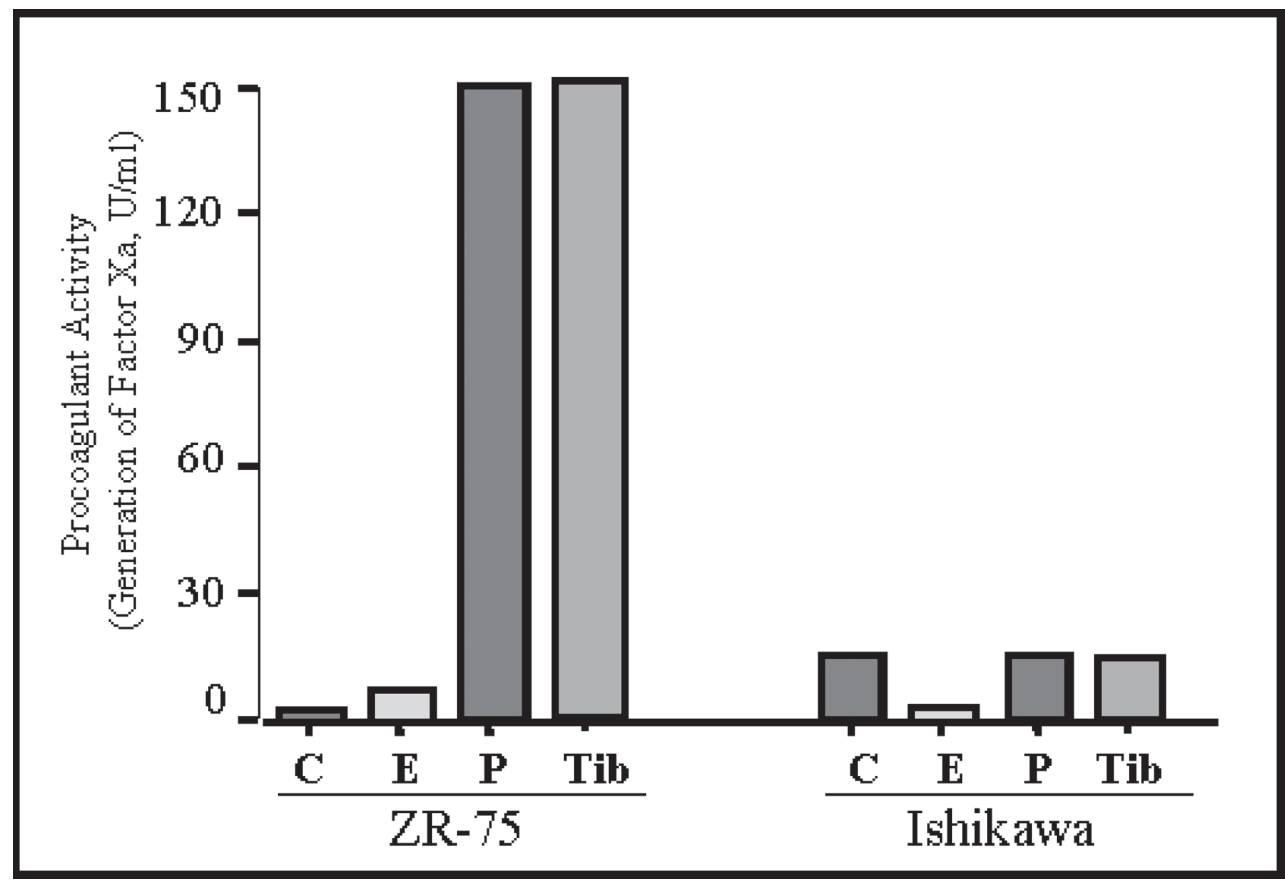

Figure 9. Procoagulant assay Effect of ovarian hormones and Tibolone on the induction of TF activity in ZR-75 and Ishikawa cells. ZR-75 and Ishikawa cells were treated with either Ethanol(C), 17- $\beta$-estradiol (E), Progesterone (P) or Tibolone (T) for $24 \mathrm{hrs}$. Procoagulant activity was measured by the formation of Factor $\mathrm{Xa}$ in the presence of Factor VIIa.

endometrial thickness with Tibolone therapy. Endometrial biopsies of women treated with Tibolone $(2.5 \mathrm{mg} /$ day) have shown an endometrial histology closely mimicking the natural atrophic postmenopausal state (27), but other studies have also found a small percentage of women in which a change from an atrophic endometrial pattern to a weakly proliferative pattern occurred (28). This indicates that Tibolone may possess both estrogenic and progestagenic properties. It has been demonstrated that transvaginal ultrasonography of the endometrium reliably predicts the histological picture. In the present study only $10 \%$ of the women showed an endometrial width over $5 \mathrm{~mm}$ indicating no hyperplastic changes in vivo with Tibolone treatment. Nevertheless, Tibolone does have an effect at the 
endometrial level, which can also be inferred due to an increase in vaginal bleeding observed in early postmenopausal women treated with Tibolone (vs. control). Bleeding was shown to be independent from endometrial stimulation (29). These data support our hypothesis that Tibolone displays estrogenic and progestagenic behavior.

In the clinic, and shown herein, it has been observed that Tibolone displays both estrogenic and progestagenic behavior. To investigate if this behavior is manifested at the cellular level, we chose to study this effect in well differentiated breast and endometrial cancer cell lines. Cell lines of breast and endometrial origin were chosen as they are important target tissues in determining the safety of potential HRT preparations. Our objective was to determine if Tibolone can regulate protein and mRNA expression in an in vitro setting, in a manner similar to estrogen and progesterone. Although the study of protein expression patterns from only two cell lines cannot deliver conclusive data on the nature of Tibolone action, we feel that this model provides evidence of the differential steroidogenic properties that Tibolone manifests in cells originating from different tissues. As depicted in the results section and summarized in Table I, Tibolone displays differential effects in our model system, acting as an estrogen or a progestin in a gene (protein)- and cell-specific manner.

\section{TABLE I}

Tibolone displays differential effects in our model system, acting as an estrogen or a progestin in a protein- and tissue-specific manner.

\begin{tabular}{lcc}
\hline & $\begin{array}{c}\text { Breast } \\
\text { (ZR75/MCF7) }\end{array}$ & $\begin{array}{c}\text { Endometrium } \\
\text { (Ishikawa) }\end{array}$ \\
\hline STAT5a/b & $\mathrm{P}$ & $\mathrm{NR}$ \\
Tissue factor & $\mathrm{P}$ & $\mathrm{NR}$ \\
Estrogen Receptor & $\mathrm{E}$ & $\mathrm{ND}$ \\
EGFR & $\mathrm{P}$ & $\mathrm{E}$ \\
Bcl-xL & $\mathrm{E}$ & $\mathrm{NR}$ \\
\hline
\end{tabular}

$\mathrm{NR}=$ No regulation $\mathrm{ND}=\mathrm{Not}$ determined $\mathrm{E}=$ estrogenic behavior; $\mathrm{P}=$ progestagenic behavior.
The estrogen receptor (ER), the target of antiestrogen breast cancer treatments such as Tamoxifen and Raloxifen (30), is rapidly down-regulated by estrogen treatment in ZR-75 cells. However, this down-regulation is transient and, after 30 hours, ER levels have risen above basal levels and are 25\% higher at 24 hours than cells which were never exposed to estrogen. Tibolone in this system acted as an estrogen. In fact, Tibolone acted as a more potent estrogen, maintaining ER at below basal levels out to the final point of this experiment at 48 hours. The down-regulation of ER, which involved in breast cancer proliferation, has potentially good implications for Tibolone as an alternative HRT treatment. While the lowering of ER levels may be seen as a beneficial effect on breast cancer risk, the increase in EGFR could be construed as a negative consequence. EGFR is overexpressed in breast cancers and is associated with a more aggressive phenotype and thus poorer patient prognosis (12).

The regulation of EGFR by Tibolone appears to mimic that of progesterone in the ZR-75 cell line and estrogen in the Ishikawa cell line. This result demonstrates the cell line-specific and possibly tissuespecific nature of Tibolone and may reflect differential metabolism between the two tissues, resulting in more estrogenic or progestagenic metabolites (discussed latter). Interestingly, it is not only Tibolone that shows this tissue-specific effect. Progesterone up-regulates EGFR expression in the cells of breast origin, but not in cells pertaining to the endometrium. This may reflect the differing nature that progesterone possesses in the differentiative preparation of the endometrium for implantation, as opposed to a more proliferative role in mammary gland ductal development. STAT5, formally known as Milk Factor, is up-regulated by progesterone in breast cell lines as anticipated. Interestingly, STAT5 is not regulated by progesterone in the endometrial cells, thus demonstrating once again the tissue-specific nature of this hormone. Tibolone acts as a progestin in the regulation of STAT5, up-regulating 
both forms of this protein in a manner similar to that of progesterone. The nature of STATs, as signal tranducers, makes a conclusion on the clinical implications of STAT5 regulation by Tibolone impossible, as STAT5 plays a vital role in mammary development and maintenance, while also being a protein which is overexpressed and possibly utilized in growth factor signaling in breast cancers (31). For example, STAT family DNA binding activity is low in normal 'resting' breast and benign lesions, while more aggressive tumors samples have significantly higher amounts of STAT binding activity (32).

Bcl-xL was chosen for this study to represent an anti-apoptotic protein. While progesterone had no effect on the regulation of this protein in either cell line studied, estrogen increased expression of this protein in both cell lines, presumably favoring a more proliferative state for these cells. Interestingly, Tibolone acted in a similar manner to estrogen by up-regulating Bcl-xL expression in the ZR-75 breast cancer cell line, while showing no regulation in Ishikawa cells. It is important to note that the process of apoptosis is a delicate balance between a host of pro-and anti-apoptotic proteins, and that a conclusion based on the regulation of one protein would be ill-advised.

TF over-expression has many clinical implications. As the initiator of the extrinsic coagulation pathway, TF has been shown to play a role in cardiovascular disease (33) and in a cancer-associated increase in the coagulative state $(34,35)$. TF also plays a role in cell invasion and cancer cell metastasis, along with possessing proangiogenic properties. Tibolone mimics the action of progesterone by up-regulating $\mathrm{TF}$ in ZR-75 cells, while no steroid treatment resulted in changes in TF expression in Ishikawa cells. To investigate this further we performed a time course in ZR-75 of Tibolone and progesterone induction of TF. As observed when Tibolone displayed estrogenic activity in the down-regulation of the ER, Tibolone again acted as a potent steroid (on this occasion acting as a progestin), maintaining TF levels elevated to the end of the experiment at 48 hours.
RT-PCR analysis demonstrated that the regulation of $\mathrm{TF}$ by both Tibolone and progesterone is occurring at the level of RNA, however transcription control or mRNA stability has yet to be determined. Given the previously reported in vitro data associating TF expression with breast cancer survival, this up-regulation of TF by Tibolone may, in part, contribute to the cancer risk conferred by Tibolone in the Million Women Study $(26,3)$.

Tibolone is known to be metabolized in a tissue-selective manner to three steroids that collectively have weak estrogenic, progestagenic, and androgenic activities (36). As alluded to in the results section, preliminary experiments in these cell lines have demonstrated that steroid metabolism occurs in these cultured cells, with estrogen and progesterone possessing a half-life in culture medium of approximately five hours. This suggests, although by no means guarantees, that these cells have the enzymes and the machinery capable of metabolizing Tibolone to its known array of active metabolites. While it would be tempting to speculate that the differential activity of Tibolone between cells of breast and endometrial origin is due to preferential metabolism into estrogenic or progestagenic metabolites, the explanation may be more complex. Estrogen and progesterone, known to bind and exert their activities in their native forms, also display cell-specific effects. in these cell lines. This may suggest the transcriptional machinery involved in steroidal mediated transcription is different between the two cell types. However, recent publications on the distribution of known steroid nuclear receptor cofactors have not provided any supportive evidence for this theory (37). Many other explanations exist. Signaling for degradation of the ER and PR may be different in the presence of Tibolone and its metabolities than that of the native ligands. Other explanations may include the formation of a more stable and higher affinity DNA binding complex in the presence of Tibolone. Furthermore, the configuration of the ligand-receptor complex is different to that of steroid compounds and thus this complex may associate to a varying degree or with 
different cofactors, thus producing a stronger or more prolonged signal. Tibolone may also produce non-transcriptional effects, possibly enhancing the stability of proteins such as $\mathrm{TF}$ and thus maintaining the elevated protein levels that are observed in Figure 6.

To verify that the Tibolone-mediated increase in $\mathrm{TF}$ converts to an increase in activity of this protein, implying that Tibolone treatment infers a physiological consequence, we tested the procoagulant activity of hormonal-and Tibolone- treated cell extracts in ZR-75 and Ishikawa cells. As anticipated, by the increase in protein observed by western blotting, both Tibolone and progesterone increased the procoagulant activity of ZR-75 cells while no changes were observed in Ishikawa cells. As we are using a cancer cell line model we cannot extrapolate this result to infer an increase in coagulation in the healthy mammary epithelium upon Tibolone treatment. However, these results suggest that treatment of a patient with Tibolone or progesterone may result in a procoagulant state.

\section{CONCLUSION}

The Million Women Study demonstrated that Tibolone increased the relative risk of both breast and endometrial cancer (3). Herein, we demonstrate that Tibolone possesses differential estrogenic and progestagenic activity in both in vivo and in vitro settings. It has been speculated that progesterone may not solely be involved in breast cancer genesis, but may also increase specific proteins, such as EGFR and TF, which provide a survival advantage to bourgeoning cancer cells, thus increasing the incidence of breast cancer in women taking progestin-containing hormonal preparations (26). This study demonstrates that Tibolone displays progestagenic activity in the up-regulation of known oncogenes such as EGFR and TF, which could be interpreted as an undesirable effect on breast cancer risk and account for the findings by the Million Women Study collaborators. However, in the clinical portion of this study Tibolone effectively reduced climacteric complaints, while in vitro, Tibolone reduced ER to below basal levels in the ZR-75 breast cancer cell line for prolonged periods of time which could be construed as beneficial to women at high breast cancer risk. Further work is needed to determine the implications of estrogen and progesterone regulation of these and other proteins involved in cancer progression. Although, in the future, Tibolone may be the single hormonal treatment which delivers both the beneficial estrogenic activities along with the progestin-mediated protection of the endometrium, the authors recommend caution and vigilance in the prescription of Tibolone and advise strict subsequent patient monitoring.

This work was supported by:

FONDECYT Project No. 1020715 (G.I.O), Wellcome Trust GR071469 (G.IO) Fundación Andes C-13685 (G.I.O) and Gynopharm (PV).

\section{REFERENCES}

1. GAMBRELL RD (1982) The menopause: benefits and risks of estrogen-progestogen replacement therapy. Fertil Steril 37(4):457-74

2. AHLBORG HG, JOHNELL O, TURNER CH (2003) Bone loss and bone size after menopause. Engl J Med 349(4):327-34

3. BERAL V, BULL D, REEVES G; Million Women Study Collaborators. (2005) Endometrial cancer and hormone-replacement therapy in the Million Women Study. Lancet. 365(9470): 1543-51

4. GOMPEL A, CHAOUAT M, Jacob D (2002) In vitro studies of tibolone in breast cells. Fertil Steril 78(2):351-359

5. EDERVEEN AG, KLOOSTERBOER HJ (1999). Tibolone, a steroid with a tissue-specific hormonal profile, completely prevents ovariectomy-induced bone loss in sexually mature rats. J Bone Miner Res 14(11):1963-1970

6. CASTELO-BRANCO C, VICENTE JJ, FIGUERAS F, SANJUÁN A, MARTÍNEZ de OSABA MJ, CASALS E, PONS F, BALASCH J, VANRELL JA (2000) Comparative effects of estrogens plus androgens and tibolone on bone, lipid pattern and sexuality in postmenopausal women. Maturitas 34(2):161-8

7. TASKIN O, MUDERRISOGLU H, AKAR M, SIMSEK M, MENDILCIOGLU I, KURSUN S (2004) Comparison of the effects of tibolone and estrogen replacement therapy on echocardiographic basic cardiac functions in post-menopausal women: a randomized placebo controlled study. Maturitas 48(4):354-9 
8. VAN DE VEN J, DONKER GH, SPRONG M (2002) Effect of tibolone (Org OD14) and its metabolites on aromatase and estrone sulfatase activity in human breast adipose stromal cells and in MCF-7 and T47D breast cancer cells. J Steroid Biochem Mol Biol 81(3):237-47

9. OWEN GI, ZELENT A (2000) Origins and evolutionary diversification of the nuclear receptor superfamily. Cell Mol Life Sci 57(5):809-27

10. OMOTO Y, EGUCHI H, YAMAMOTOYAMAGUCHI Y (2003) Estrogen receptor (ER) beta1 and ERbetacx/beta2 inhibit ERalpha function differently in breast cancer cell line MCF7. Oncogene 22(32):5011-20

11. YAMASHITA H, IWASE H, TOMOYAMA T (2003) Naturally occurring dominant-negative Stat5 suppresses transcriptional activity of estrogen receptors and induces apoptosis in T47D breast cancer cells Oncogene 20; 22(11):1638-52

12. SOLBACH C, ROLLER M, AHR A (2002) Antiepidermal growth factor receptor-antibody therapy for treatment of breast cancer. Int J Cancer 1;101 (4):390-4

13. HANANHAN D, WEINBERG RA (2000) The hallmarks of cancer.Cell. 100(1):57-70

14. KONINGSBERG W, KIRCHHOFER D, RIEDERER M (2001) The tissue factor:VIIa: clinical significance, structure function, relationships and its role in signaling and metastasis. Tromb and Haemost 86(5):757-71

15. UENO T, TOI M, KOIKE M (2000) Tissue factor expression in breast tissue: its correlation with prognosis and plasma concentration. Br J. Cancer 83(2):164-70

16. VRANA JA, STANG MT, Grande JP (1996) Expression of tissue factor in tumor stroma correlates with progression to invasive human breast cancer: paracrine regulated by carcinoma cell-derived members of the transforming growth factor beta family. Cancer Res 56(21):5063-70

17. SHOJI M, HANCOCK W, ABE M (1998) Activation of coagulation and angiogeneis in cancer: Inmunohistochemical localization in situ of clotting proteins and vascular endothelial growth factor in human cancer. Am J Pathol 52(2):399-411

18. SORENSEN BB, RAO LV, TORNEHAVE D (2003) Anti-apoptotic effect of coagulation factor VIIa. Blood 102(5):1708-15

19. SUBRAMANIAN T, CHINNNADURAI G (2003) Proapoptotic activity of transiently expressed BCL-2 occurs independent of BAX and BAK. J Cell Biochem $15 ; 89(6): 1102-14$

20. ENGEL LW, YOUNG NA, TRALKA TS (1978). Establishment and characterization of three new continuous cell lines derived from human breast carcinomas. Cancer Res 38(10):3352-64

21. HORWITZ KB, COSTLOW ME, McGUIRE WL (1975) MCF-7; a human breast cancer cell line with estrogen, androgen, progesterone, and glucocorticoid receptors. Steroids 26(6):785-95.

22. NISHIDA M, KASAHARA K, KANEKO M (1985) Establishment of a new human endometrial adenocarcinoma cell line, Ishikawa cells, containing estrogen and progesterone receptors. Nippon Sanka Fujinka Gakkai Zasshi 37(7):1103-1111
23. CHOMCZYNSKI P, SACCHI N (1987) Single-step method of RNA isolation by acid guanidinium thiocyanate-phenol-chloroform extraction. Anal Biochem 162(1):156-159

24. SEEGER H, WALLWIENER D, MUECK AO (2003) The effect of progesterone and synthetic progestins on serum- and estradiol-stimulated proliferation of human breast cancer cells Horm Metab Res 35(2):76-80

25. HATA H, KURAMOTO H (1992) Immunocytochemical determination of estrogen and progesterone receptors in human endometrial adenocarcinoma cells (Ishikawa cells). J Steroid Biochem Mol Biol 42(2):201-210

26. KATO S, PINTO M, CARVAJAL A, ESPINOZA N, MONSO C, SADARANGANI A, VILLALÓN M, BROSENS JJ, WHITE JO, RICHER JK, HORWITZ KB, OWEN GI (2005) Progesterone increases Tissue Factor gene expression, procoagulant activity and invasion in the breast cancer cell line ZR-75-1. J Clin Endocrinol Metab 90(2):1181-8

27. HUBER J, PALACIOS S, BERGLUND L (2002) Effects of tibolone and continuous combined hormone replacement therapy on bleeding rates, quality of life and tolerability in postmenopausal women. BJOG 109(8):886-93

28. VOLKER W, COOELINGH BENNINK HJ, HELMOND FA (2001) Effects of tibolone on the endometrium. Climacteric 4(3):203-8

29. BERNING B, van KUIJK C, BENNINK HJ (2000) Absent correlation between vaginal bleeding and estradiol levels or endometrial morphology during tibolone use in early postmenopausal women. Maturitas 35(1):81-8

30. BARKER S (2003) Anti-estrogens in the treatment of breast cancer: current status and future directions. Curr Opin Investig Drugs 4(6):652-657

31. YAMASHITA H, IWASE H (2002). The role of Stat5 in estrogen receptor-positive breast cancer. Breast Cancer 9(4):312-8

32. SILVA CM (2004) Role of STATs as downstream signal transducers in Src family kinase-mediated tumorigenesis. Oncogene 3(48):8017-8023

33. DAHM A, Van HYLCKAMA VLIEG A, BENDZ B (2003) Low levels of tissue factor pathway inhibitor (TFPI) increase the risk of venous thrombosis. Blood 101(11):4387-92

34. MEDINA R, ARANDA E, VERDUGO C, KATO S, OWEN GI 2003 The action of ovarian hormones in cardiovascular disease. Biol Res 36: 325-341

35. KATO S, PINTO M, CARVAJAL A, ESPINOZA N, MONSO C, BRAVO L, VILLALÓN M, CUELLO M, QUEST A.F., SUENAGA A, BROSENS JJ., OWEN GI. (2005). Tissue factor is regulated by epidermal growth factor in normal and malignant human endometrial epithelial cells. Thrombosis and Haemostasis., 94 (2) 444-453

36. KLOOSTERBOER HJ (2001). Tibolone: a steroid with a tissue-specific mode of action. J Steroid Biochem Mol Biol 76(1-5):231-238

37. GRAHAM JD, BAIN DL, RICHER JK, JACKSON TA, TUNG L, HORWITZ KB (2000). Thoughts on tamoxifen resistant breast cancer. Are coregulators the answer or just a red herring? J Steroid Biochem Mol Biol 30;74(5):255-9. 